\title{
Synthesis and characterization of the fluorescentself-assembled structures formed by Benzothiazolone conjugates and applications in cellular imaging
}

VivekshinhKshtriya $^{[\mathrm{a}]}$, Bharti Koshti $^{[\mathrm{a}]}$, AnkitGangrade ${ }^{[\mathrm{b}]}$, Ashadul Haque ${ }^{[\mathrm{b}]}$, Ramesh Singh

Chilwal, KB Joshi, Dhiraj Bhatia, ${ }^{[\mathrm{b}] *}$ Nidhi Gour ${ }^{[\mathrm{a}]}{ }^{*}$

[a] Department of Chemistry, Indrashil University, Kadi, Mehsana, Gujarat, India E-mail: nidhigour@gmail.com

[b] Biological Engineering Discipline and Center for Biomedical Research, Indian Institute of Technology Gandhinagar, Palaj 382355, Gandhinagar, India E-mail: dhiraj.bhatia@iitgn.ac.in

[c] Department of Chemistry, Dr. Hari Singh Gour, Sagar University, Madhya Pradesh, India

Abstract: We report the synthesis and characterization of self-assembled structures formed by4-Choro-2(3H)-benzothiazolone (VK) to panchromatic fibers and its application as cell imaging tool. The aggregation properties ofthe synthesized compounds have been studied extensively under different solvent and concentrationand theirmorphologies examined at supramolecular level was observed by microscopic techniques like optical microscopy, fluorescence microscopy, and atomic force microscopy(AFM). Interestingly, the selfassembled structures formed by VKreveal panchromatic emission properties andshow blue, green and red fluorescence under different excitation filters. The intensity of the fluorescence observed was blue>green>red and the dye interestingly do not show any fluorescence quenching, on the other hand reveal photoactive properties under green channel. The mechanisms of formation of the self-assemblies were studied through different techniques like concentration dependent NMR and,UV visible spectroscopy and fluorescencemicroscopic studies.Finally, the utility of VK for cell imaging applications is 
demonstrated and it can be noted that VK can be efficientlyup taken by mammalian cells and the stained cells reveal panchromatic emission under blue, green and red channel.

\section{Introduction:}

Molecular self-assemblyisconsidered asvery fast-growing field of research considering its crucial significance in the field of material science, ${ }^{1-4}$ chemistry ${ }^{3,5,6}$ and biological science ${ }^{3,7,8}$ for diverse applications. The study of molecular assembly of any compound is important since their organization at supramolecular level plays a decisiverole for ascertaining their potential applications as in drug delivery and sensing ${ }^{9-10}$

The main forces responsible for the formation of self-assembled structures arethe noncovalent interactions like vander Waal's, hydrogen bonding and pi-pi stackingwhich results in formation of well-defined morphologies throughbottom-up approach. ${ }^{8-10}$ Moreover, the self-assembling properties of the compound also influences its photophysical characteristics. ${ }^{11-13}$ which include its conductivity, ${ }^{14-16}$ optoelectronic properties ${ }^{17,}{ }^{18}$ and its dispersion capability, ${ }^{19,}{ }^{20}$ as well as the lattice packing of the molecules. ${ }^{21,}{ }^{22}$ The selfassembling properties of the material is also affected by the various parameters such as concentration, ${ }^{23,}{ }^{24}$ temperature, $\mathrm{pH},{ }^{25,}{ }^{26}$ solvent, $^{27,}{ }^{28}$ and lattice arrangement. ${ }^{29-31}$ The importance ofthe self-assembly/aggregation on the photophysical properties of the material can be broadly understood by two terminologiesaggregation induced emission ${ }^{34}$ and aggregation caused quenching ${ }^{35}$.

We have earlier demonstrated synthesis and characterization of biomolecular self-assemblies and its applications as sensing and drug delivery tools. ${ }^{36-41}$ Recently, our group reported a new pyridothiazole based AIEE probe for sensitive detection of amyloids. ${ }^{42}$ Herein, we report the self-assembly of 4-Choro-2(3H)-benzothiazolone (VK)to fibers exhibiting panchromatic 
emission. The self-assembly studies are done under varying solvent and concentration and the mechanism of its formation assessed by NMR and UV visible spectroscopy, Fluorescence spectroscopy.Notably, VK is an intermediate which iswidely used for the preparation of benazolin-ethyl, a well-knownherbicide used in agriculture.It has immense applications in pharmaceutical and agrochemical industries, agrochemical some of the noted products based on VK scaffold being Chlobenthiazone, Benazolin-ethyl, \pm )-Mevashuntin..$^{32}$ to name a few. Hence, the study pertaining to aggregation and photophysical characteristic of this benzothiazolone conjugate is particularly significant for assessing its implications in drug delivery and sensing.

\section{Results and discussions}

VK was synthesised in five steps through already reported synthetic methodologies. ${ }^{32}$. In the first step condensation reaction is carried out between 2-chloraniline and benzoyl isothiocyanate. The step is followed by alkaline hydrolysis which yields 2-chlorophenyl thiourea. Further, oxidative cyclization reaction is carried out using $\mathrm{Br}_{2}$ to yield 4chlorobenzo[d]thiazol-2-amine. In the next step, diazotization reaction was carried out to yield 2,4-dichlorobenzo[d]thiazole. Finally, refluxing with concentrated $\mathrm{HCl}$ is pursued to yield VKin good quantitative as well as qualitative yield via Scheme 1.

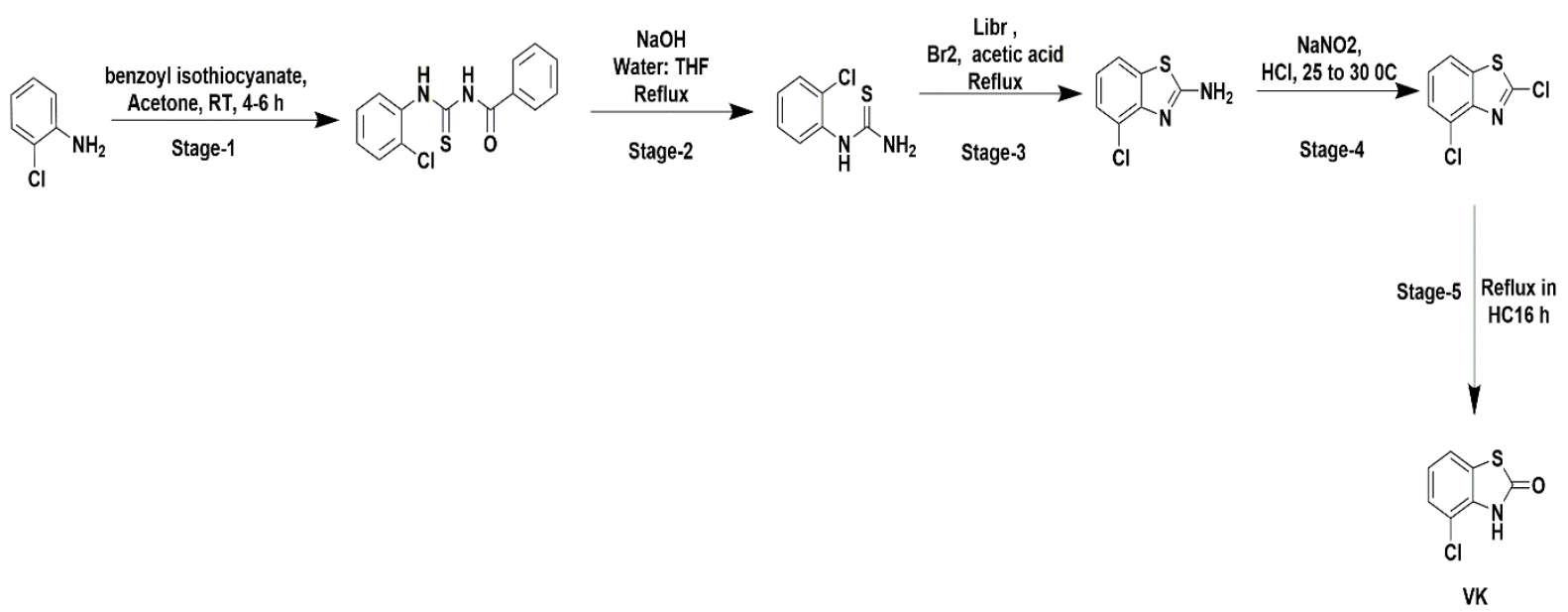


Scheme 1 Chemical structure and synthesis of4-Choro-2(3H)-benzothiazolone.

The synthesized VK was characterized by ${ }^{1} \mathrm{H}$ and ${ }^{13} \mathrm{C}$ NMR spectroscopy and LCMS mass analysis. The purity of VK was ascertained by HPLC. After achieving sufficient characterization and purity, the self-assembling properties of VK were analysed in water at various concentrations.

The self-assembly studies of VK were done by dissolving VK in DMSO to make a stock solution of $50 \mathrm{mM}$. The solution was subsequently diluted to $1 \mathrm{mM}$ by dissolving it in deionized water. The self-assembling behaviour of VK (1mM) was studied by atomic force microscopy (AFM). AFM analysis reveals VK assemble to fibers which also show branching (Figure 1). The fibers were having diameters in microns range and their length ranged to several micrometers. The fibers were soft in nature as can be assessed by the AFM tip force when recorded in tapping mode.
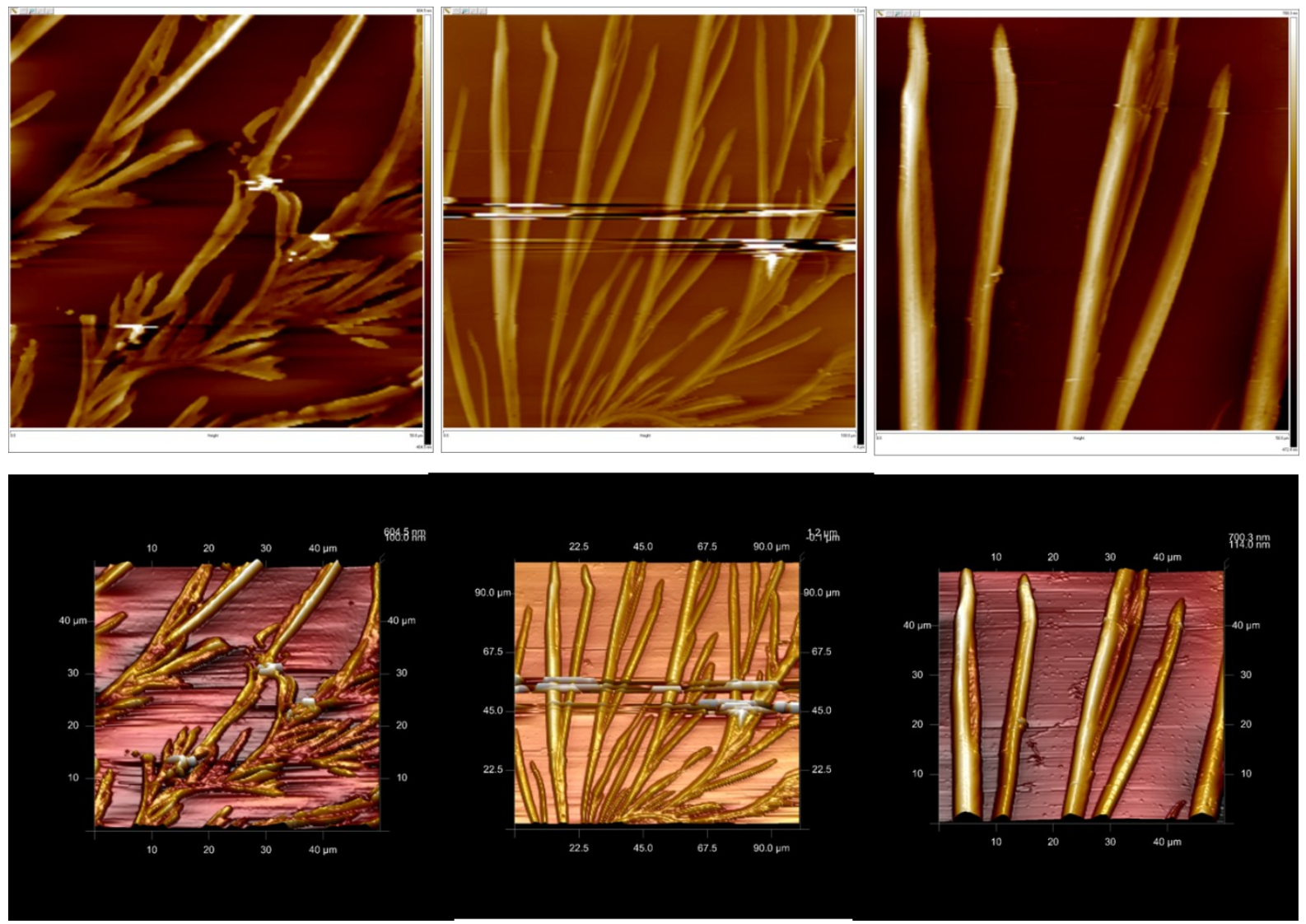

Figure 2 Atomic ForceMicroscopy (AFM) images of self-assembled structure $\mathbf{V K}(1 \mathrm{mM})$ in 2\% DMSO water solution under different magnifications. 
The fibres of VK could also be observed by simple optical microscopy since their dimensions were large. A concentration dependent analysis of fiber morpholgu was done using optical and fluorescence microscopy at $1 \mathrm{mM}, 3 \mathrm{mM}, 5 \mathrm{mM}$ and $7 \mathrm{mM}$ concentrations As the concentration is increased fibers appear to become more thick and form tape like structure at high concentrations. Interestingly, under green filter these fibers also reveal intense fluorescence without the use of any dye in fluorescence microscopy. Hence, it may be inferred that $\mathrm{VK}$ is fluorescent in nature and hence assemble to fluorescent fibers. Interstinngly, when the fibers were observed in red filter a slight fluorescence with relatively less intensity than green channel could be observed which suggested VK might exhibit panchromatic fluorescence.
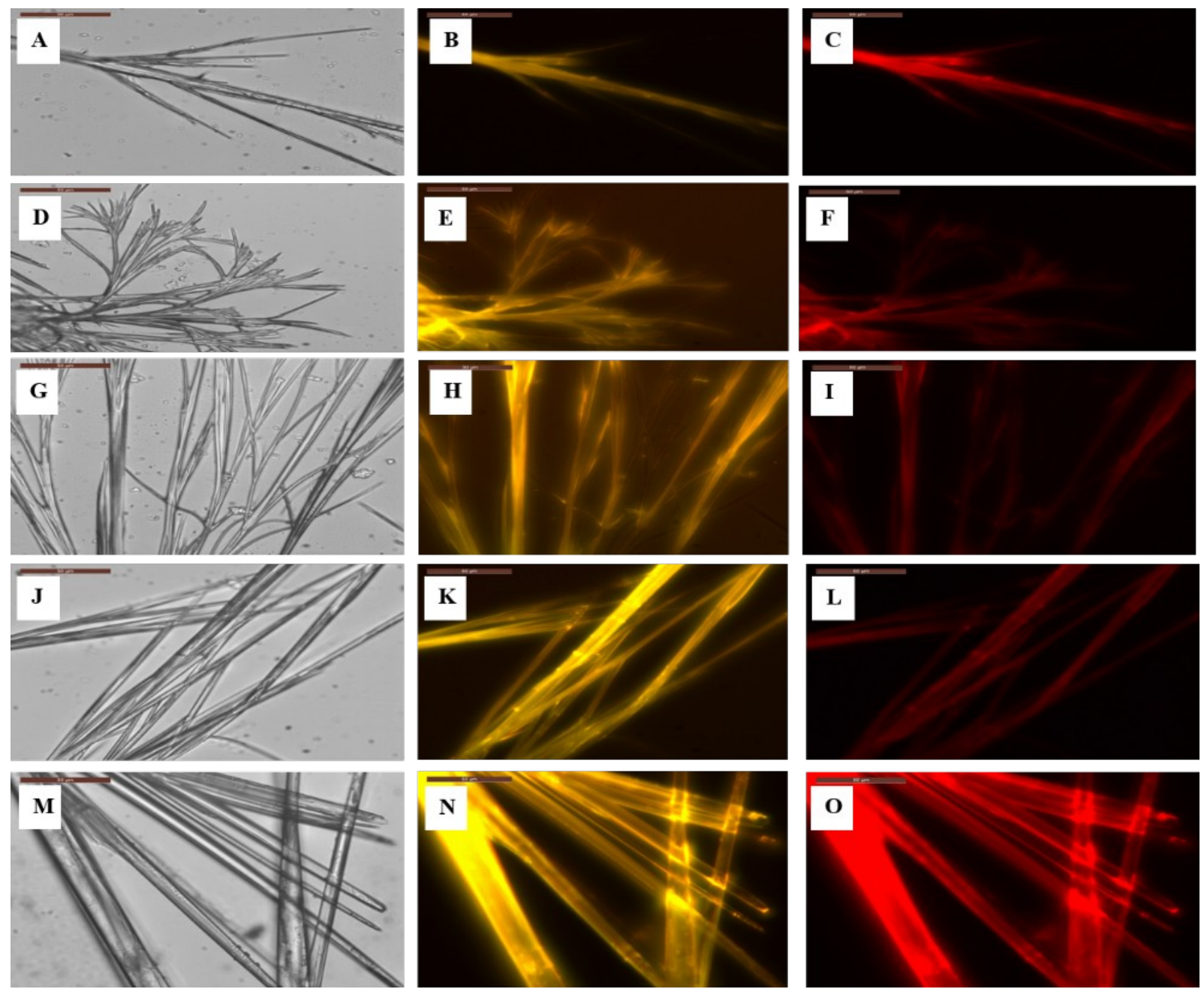
Figure 2. Microscopy images of self-assembled structure VK in 2\% DNSO: Water.; A) $1 \mathrm{mM}$ concentration under bright field; B) $1 \mathrm{mM}$ concentration under green filter; C) $1 \mathrm{mM}$ concentration under red filter; D) $3 \mathrm{mM}$ concentration under bright field; E) $3 \mathrm{mM}$ concentration under green filter; F) 3mM concentration under red filter; G) $5 \mathrm{mM}$ concentration under bright field; H) $5 \mathrm{mM}$ concentration under green filter; I) $5 \mathrm{mM}$ concentration under red filter; J) $7 \mathrm{mM}$ concentration under bright field; K) $5 \mathrm{mM}$ concentration under green filter; L) $7 \mathrm{mM}$ concentration under in red filter; M) $9 \mathrm{mM}$ concentration under bright field; N) $9 \mathrm{mM}$ concentration under green filter; O) $9 \mathrm{mM}$ concentration under red filter.

Hence. the panchromatic emission properties of VK were also examined in detail by fluorescence spectroscopy under various excitation wavelengths. From the fluorescence spectra it may be inferred that VK reveals best fluorescence properties in excitation wavelength@360 nm, since the emission spectra appears to be an mirror image of the excitation plot. (Figure 3a, 3b). The emission properties range from 370-610 covering most region of VIBGYPR spectrum which justifies the panchromatic emission of VK.
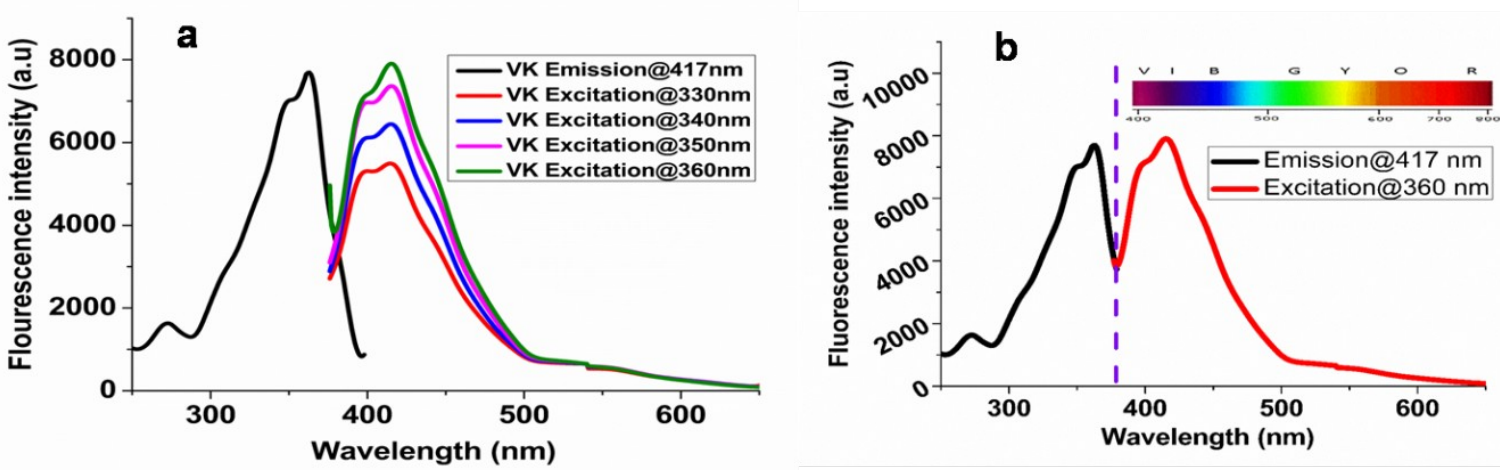

Figure 3. Fluorescence spectra of VK 1mM in 2\% DMSO: water.

Further, to understand the mechanism through which VK assemble to panchromatic fibers we resorted to concentration dependent NMR studies. These studies were done to understand the role of pi-pi stacking The study revealed that as we increased the concentration of VK, there was an upfield peak shift in the aromatic region between 7 to $9 \mathrm{ppm}$ which could be observed by the concentration dependent NMR spectra (Figure $\mathbf{4 b - 4 d ) . ~}$ 
As the concentration increases. the intensity of peak is also increased and there is a simultaneous aromatic peak shift up field, indicating increase shielding of the protons due to pi-pi stacking which causes increase in the electron density aroung aromatic nucei. As the concentration of the VK solution is increased there is enhanced pi-pi stacking and molecules are stacked more and more close causing upfield shift. ${ }^{3324,34,35}$
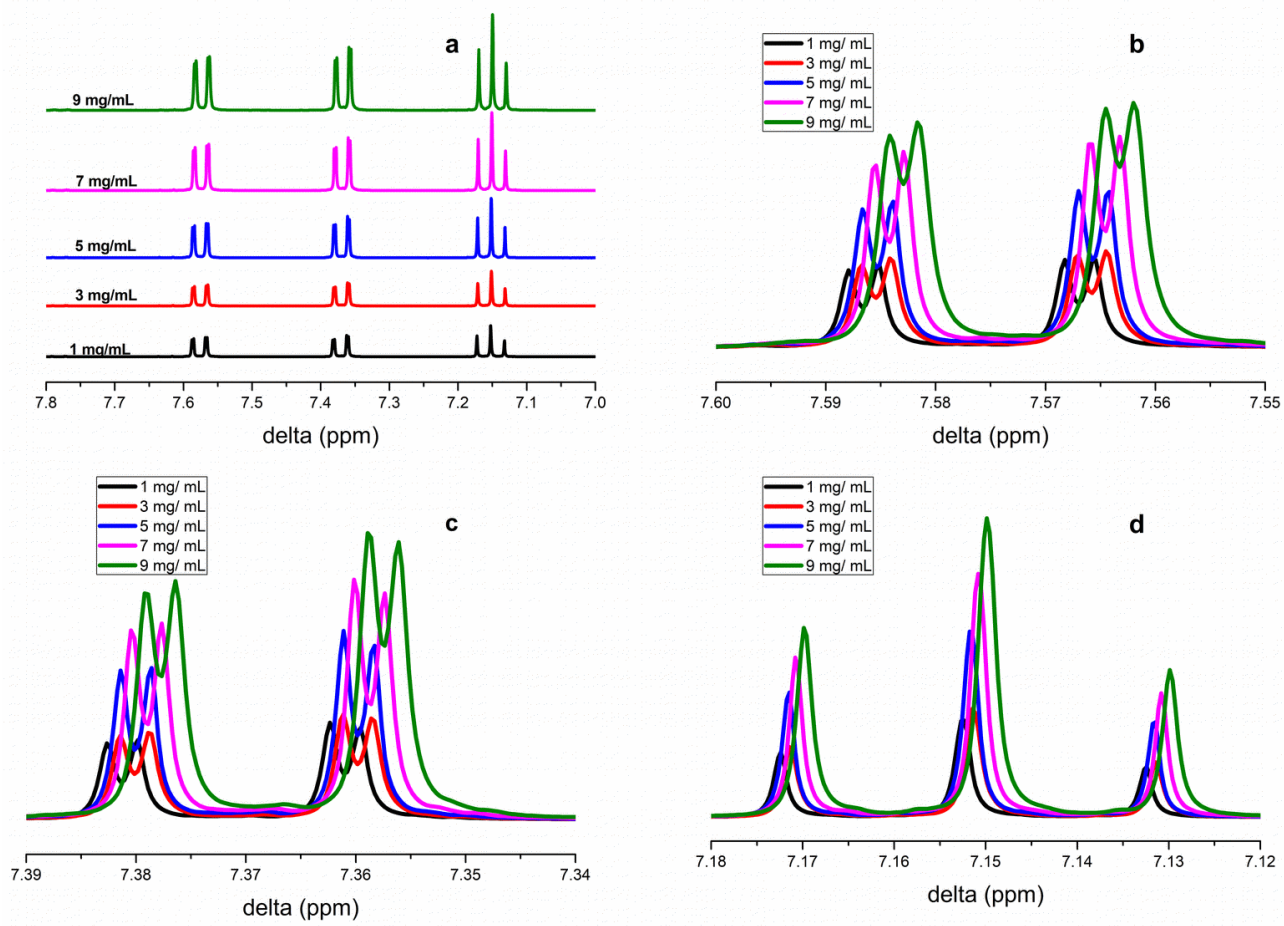

Figure 4: Concentration dependent NMR spectra of VK at 1, 3, 5, 7, $9 \mathrm{mg} / \mathrm{mL}$ in DMSOd6.Offset graph of VK1,3,5,7,9 mg/mL; b) 7.55-7.60 ppm expansion; c) 7.34-7.39 expansion; d) 1.127.18 expansion.

Further, concentration dependent UV studies were carried out in the concentration range of 5 to $200 \mu \mathrm{M}$ by using $50 \mathrm{mM}$ stock solution of VK in DMSO and diluting it appropriately by deionized water. The study revealed VK show three peaks at 285, 294 and $319 \mathrm{~nm}$ respectively (Figure 6a-6b) Further,VK obey the lambert beer; law, at wavelength 285 and $319 \mathrm{~nm}$ which is exhibited by a straight-line graph of increased absorbance with increasing \ concertation. (Figure 6c, 6d).. It was observed that as the concentration of VK was increased 
from $0 \mathrm{uM}$ to $100 \mathrm{uM}$ their was a slight red shift, Hence. we performed the concentration dependent study of VK to decipher the role of pi-pi stacking and hydrogen bonding in the self-assembled aggregated formation. Hence, we recorded the UV spectra of VK under different concentration $0.15 \mathrm{mM}, 0.20 \mathrm{mM}, 0.25 \mathrm{mM}, 0.5 \mathrm{mM}$ and $0.75 \mathrm{mM}$ (Figure 7a-7c) in different solvents like dimethyl sulphoxide, methanol and water. ${ }^{24,}{ }^{33}$ It was surmised that as the polarity of solvents will change their will be a shift in spectra which can reveal the crucial role of hydrogen bonding in the self-assembly process.
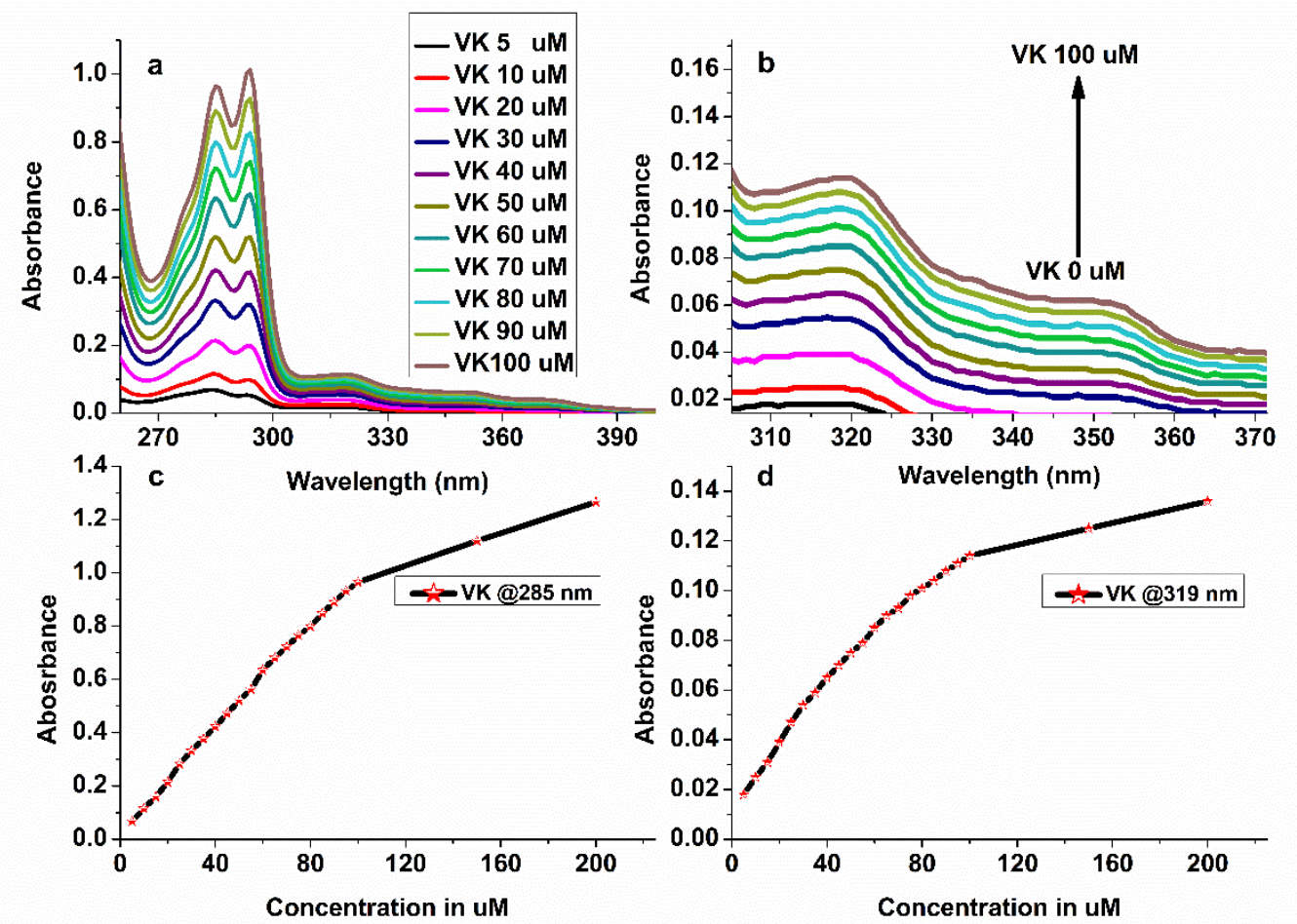

Figure 5: UV spectra of VKa) UV spectra from 5 to $100 \mathrm{uM}$; b) UV spectra expansion between 310 to 370 nm; c) straight line graph @285 nm; d) straight line graph @319 nm 
The UV visible study of VK revealed as the polarity of solvent increases their was peak broadening. The UV graph of VK, was studied in water, methanol and DMSO.
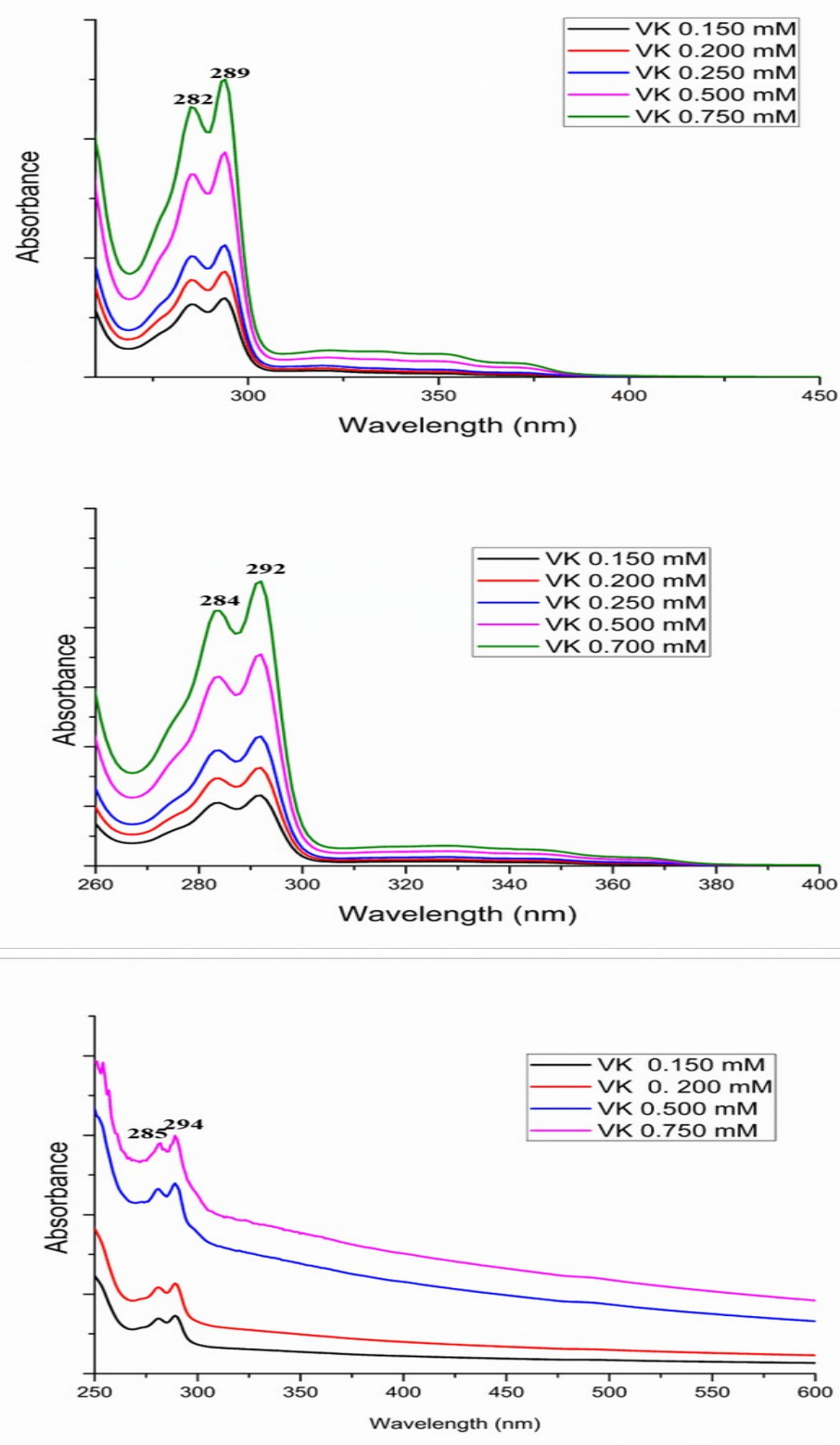

Figure 6. UV visible spectra of VK in DMSO (top); methanol (middle) and water (bottom) indicating red shift and peak broadening as the polarity is increased.

Water has maximum polarity due to its high dialectic constant followed by methanol, DMSO, As we record the UV spectra of VK in three different solvent we observed that theUV graph display two peak in between interval of $10 \mathrm{~nm}$, UV study in DMSO reveal two peaks at 282 
and $289 \mathrm{~nm}$, with a broad hump which might pointed to the presence of aggregates formed due to pi-pi stacking., When the UV spectra of VK was recorded in methanol, ,the peak at $282 \mathrm{~nm}$ observed in the case of DMSO shifts to $284 \mathrm{~nm}$ and peak at 289 shifts towards 292 nm. Further when UV spectra of VK were recorded in water there was maximum peak shift to $285 \mathrm{~nm}$ and $295 \mathrm{~nm}$ respectively, indicating as the solvent polarity is increased there is a red shift and also also more peak broadening indicating increased aggregation due to pi-pi stacking and hydrogen bonding. Further, concentration dependent microscopy studies presente in Figure 3 supports UV visible and NMR observation. More importantly, as we use non polar solvent like THF the assemblies are disrupted. This study further indicates crucial role of hydrogen bonding in the self-assembly of VK.
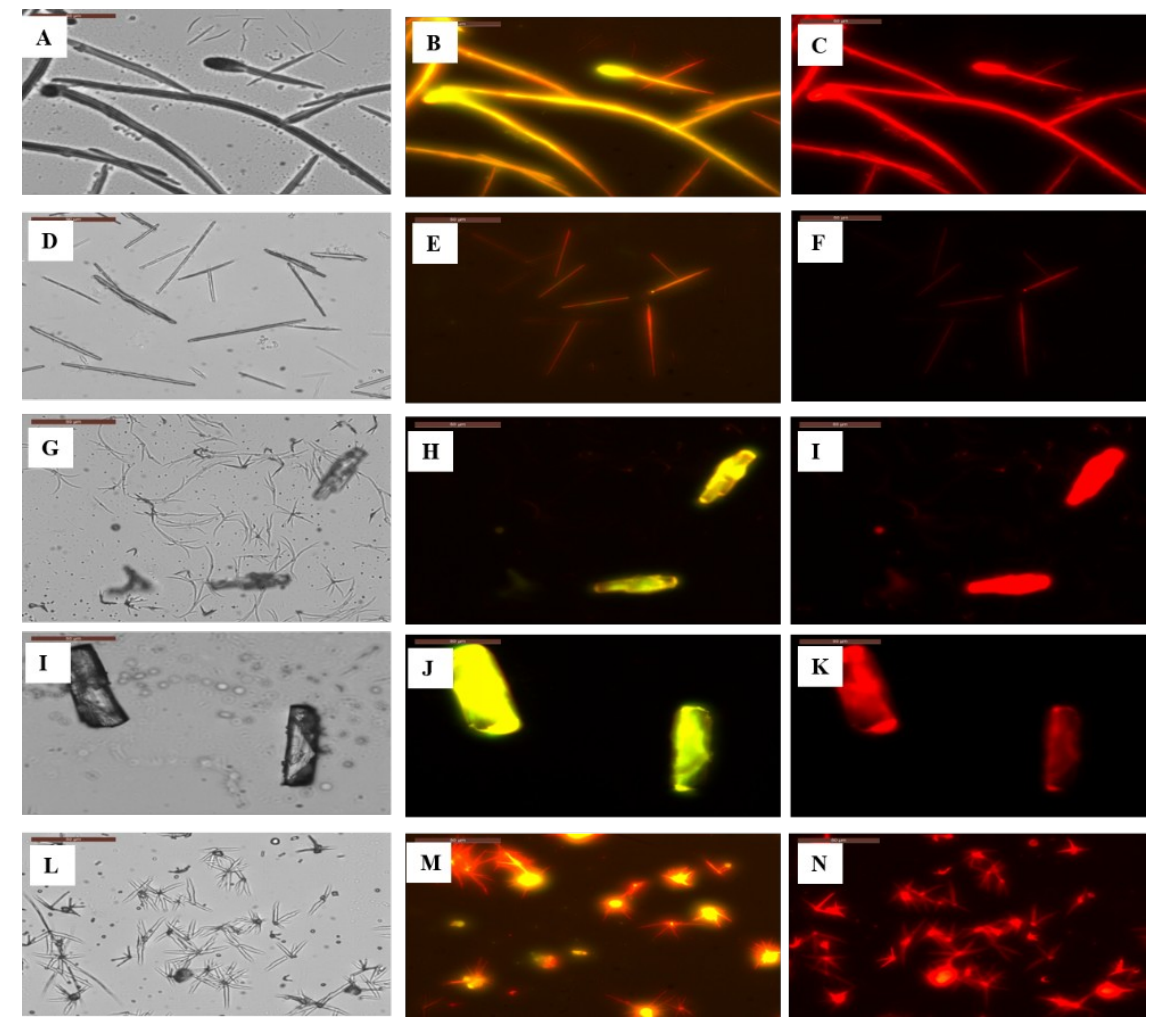

Figure 7. Microscopy images of self-assembled structure VK in THF prepared from 50mM stock in DMSO; A) $1 \mathrm{mM}$ concentration in bright field; B) $1 \mathrm{mM}$ concentration in green filter; C) $1 \mathrm{mM}$ concentration in red filter; D) $3 \mathrm{mM}$ concentration in bright field; E) $3 \mathrm{mM}$ concentration in green filter; F) $3 \mathrm{mM}$ concentration in red filter; G) $5 \mathrm{mM}$ concentration in bright field; H) 5 mM concentration in green filter; I) 5 mM concentration in red filter; J) 7 
$\mathrm{mM}$ concentration in bright field; K) $5 \mathrm{mM}$ concentration in green filter; L) $7 \mathrm{mM}$ concentration in red filter; M) 9 mM concentration in bright field; N) $9 \mathrm{mM}$ concentration in green filter; O) 9 mM concentration in red filter.

Hence, the mechanism of self-assembly of VK can be explained by pi-pi stacking and hydrogen bonding and can be represented graphically as in Figure 8.

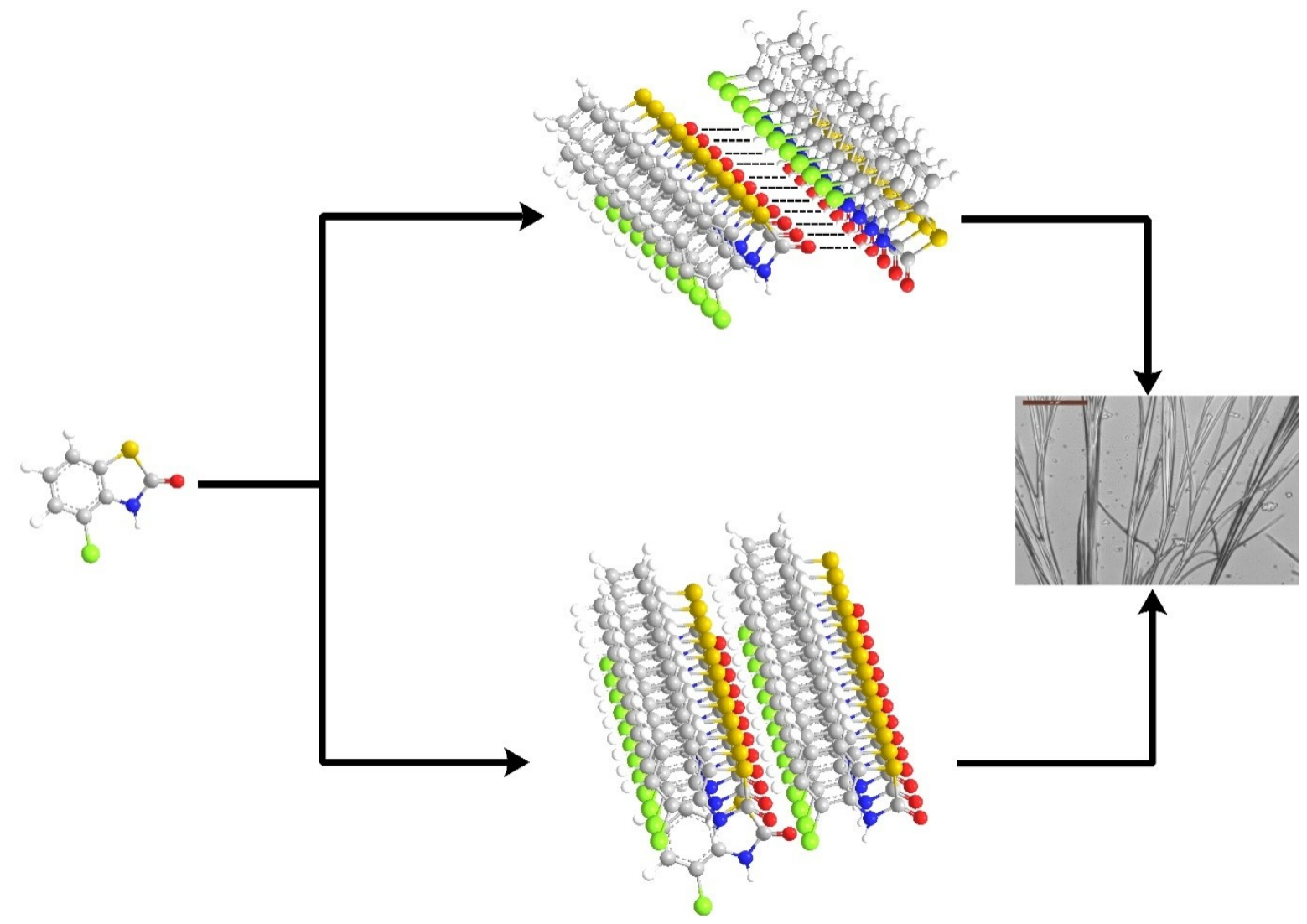

Figure 8: Graphical representation of VK self-assembled structure through the pi-pi stacking and hydrogen bonding

Hence once the mechanism of VK self-assembly was deciphered, our aim was to assess the application of VK. The panchromatic emission properties observed for VK self-assembled structures indicate their potential application as dye for bio-imaging. Hence, cellular uptake of VK was studies and the cells were imaged to identify its applications.

The fluorescence of the VK compound was concentration-dependent (Figure 9). VK showed significantly high fluorescence on excitation with $488 \mathrm{~nm}$ laser and almost negligible fluorescence on excitation with $633 \mathrm{~nm}$ laser. However, VK's fluorescence intensity on 
excitation with $405 \mathrm{~nm}$ laser was significantly increased by increasing the concentration from $500 \mu \mathrm{M}$ to $1 \mathrm{mM}$. This may be explained, since for bio imaging application confocal laser is fived at Ex of $405 \mathrm{~nm}$, while VK real Ex is $360 \mathrm{~nm}$ as discussed in Figure 3. Hence a relatively less blue fluorescence is visible since during bio-imaging the optimal excitation wavelength is changed so that there are no harm to cells due to high energy radiations, Under green filter optimal fluorescence is observed due to Ex of $488 \mathrm{~nm}$ which is used in fluresence microscopy analysis of fibers too. The fluorescence in red region was also low since here again the excitation was $633 \mathrm{~nm}$ which is different from 540-560 nm used in the florescence microscopy. Hence from the cellular imaging data it may be inferred that VK can be potentiall used as bio-imaging dye in future.
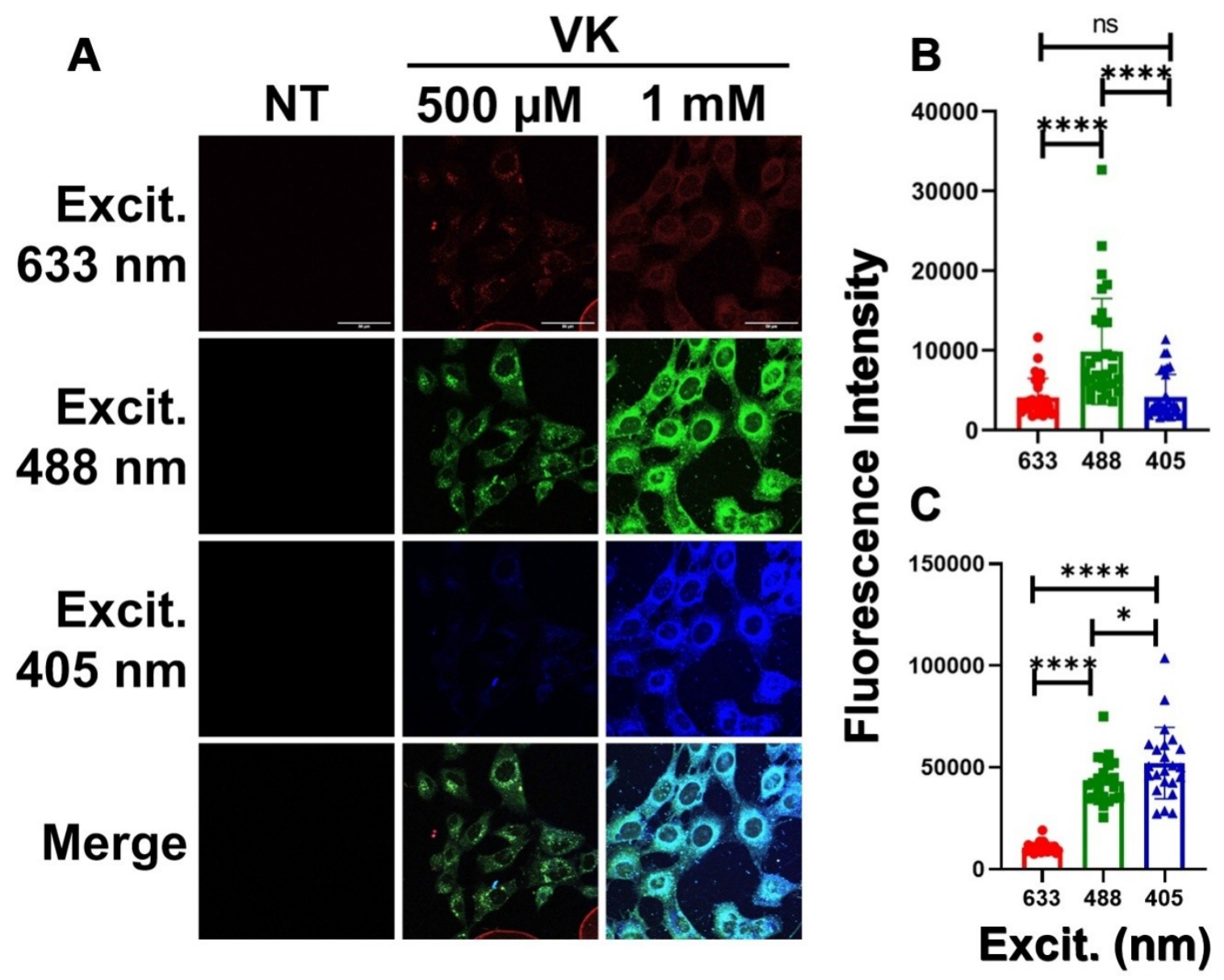

Figure 8. Cellular uptake and imaging of VK dye. Representative (A) confocal microscopy images of human breast cancer cell line (MDA-MB-231) treated with $500 \mu \mathrm{M}$ and $1 \mathrm{mM}$ 
synthesized VK compound. The VK treated cells were excited with three lasers and corresponding emission was collected as follow. (excitation $633 \mathrm{~nm}$; emission 643 to $796 \mathrm{~nm}$, excitation $488 \mathrm{~nm}$; emission 500 to $600 \mathrm{~nm}$, excitation $405 \mathrm{~nm}$; emission 415 to $500 \mathrm{~nm}$. Approximately 30 cells were selected from three different images and their fluorescence intensity was quantified. Fluorescence quantification plot at (B) $500 \mu \mathrm{M}$ and (C) $1 \mathrm{mM}$ concentration VK treated cell at all three excitation wavelengths. $* * * *$ Indicates significance level at $\mathrm{P}<0.0001, *$ indicate significance level at $\mathrm{P}<0.0329$ and ns indicates no significance between the intensities.

\section{Conclusions}

In conclusion we have reported facile synthesis and characterization of self-assembled structures formed by 4-Choro-2(3H)-benzothiazolone (VK) to panchromatic fibers and its application as cell imaging tool. VK is a commonly used intermediate used as agrochemical herbicide. The aggregation properties were studied extensively by different microscopy and spectroscopic techniques. The mechanism of self-assembly was deciphered by concentration dependent NMR and UV visible studies. Further solvent dependent studies revealed crucial affect of polarity on the self-assembled structures. Finally, the utility of VK for cell imaging applications was demonstrated and it can be observed that VK can be efficiently up taken by the mammalian cells and the stained cells reveal panchromatic emission under blue, green and red channel..

\section{Experimental}

2-Chloro aniline, Sodium thiocyanate, concentrated sulphuric acid, concentrated hydrochloric acid were purchased from merk (India), sodium bromide, acetone, sodium nitrate, purchased from spectrochem (India), all the solvent is used during experiment prior to used distilled and dry.

\section{Synthesis and characterization of VK}

VK-Stage-4 crude(7.0 g, $37 \mathrm{mM}, 1.0$ eq.) was dissolved in $36 \%$ concentrated $\mathrm{HCl}$ and heat at temperature 110 to $115{ }^{\circ} \mathrm{C}$ for 10 to 12 hours on oil bath with water condenser, The 
reaction monitor shows that the formation of small particle after hydrolysis and product formation, during the progress there was the loss of $\mathrm{HCl}$, so need to be added more $36 \%$ $\mathrm{HCl}$, monitor the TLC shows in 50\% ethyl acetate: Hexane showed that the starting material consumed, stop the heating and allow to cool the reaction mixture at room temperature again cooled the reaction mixture at 0 to $5{ }^{\circ} \mathrm{C}$ temperature and filtered the reaction mixture in the cooling then after dry the wet cake in rotavapor under reduced pressure which gives 6.5 gm crude material which was further purified by crystallization process in acetone by dissolving the crude material in acetone and reflux for 30 to 45 minutes and filtered the reaction mass at $60{ }^{\circ} \mathrm{C}$, dry the wet cake in rota vapour in reduced pressure which gives the 5 gm VK final product, the purity of VK has been confirmed by the HPLC method purification. M.P. $174{ }^{\circ} \mathrm{C}$; Rf 0.38, $1 \mathrm{H}$ NMR $\left(400 \mathrm{MHz}, \mathrm{DMSO}-\mathrm{d} 6,25^{\circ} \mathrm{C}\right.$, $\mathrm{TMS}) \delta(\mathrm{ppm})=12.20(\mathrm{~s}, 1 \mathrm{H}), 7.57(\mathrm{dd}, \mathrm{J}=8.0 \mathrm{~Hz}, 2 \mathrm{H}), 7.36(\mathrm{~d}, \mathrm{~J}=8.0 \mathrm{~Hz}, 2 \mathrm{H}), 7.16-7.12(\mathrm{t}$, 3H). LC-Mass for C7H9N3OS was calculated 185.19 and found $[\mathrm{M}+\mathrm{H}]+=186.19$

using 50mM stock of VK. prepared in DMSO, 3 The self-assembling properties of VKwere assessed first by opticalmicroscopy. In different solvents namely, Milli-Q water, THF, Methanol, Isopropanol, Ethanol, Ethyl acetate, DMF, acetone, dichloromethane, Acetonitrile, chloroform, dimethyl sulphoxide, Hexane, out of this we get fibre like assemblies in the water, DMSO, Methanol, and Ethanol and acetone further we study the self-assembly properties of VK in the range of 1 to $10 \mathrm{mM}$ (ESI 1) concentration and observed the assembly, in this paper we mainly focus on the self-assembly $\mathbf{V K}$ in water.

\section{Aggregation study of VK}

The aggregation study of VK has been study in DMSO: water mixture which deciphered that VK is itself fluorescence active in solution form in DMSO, When the fraction of water has been increased from 10 to $100 \%$, at beginning the fluorescence has been increased the till 50 
$\%$ water fraction while after $50 \%$ fraction fluorescence gradually decreased and at $90 \%$ water fraction fluorescence complete quenching observed which clearly reveal that the aggregation caused quenching phenomenon. Which also supported that the aggregation caused quenching take place due to the formation excimers in the solution, the main cause of formation of excimers due to pi-pi stacking ${ }^{36}$ which hindered the radiative emission of VK when it comes from S1 to S0 state as per Jablonski diagram.

\section{In vitro bioimaging assessment}

Human breast cancer (MDA-MB-231) cells were grown in DMEM cell culture media supplemented with 10\% FBS and 1\% antibiotic in a 5\% CO2 incubator. First, the cells were seeded onto a glass coverslip and fixed with $4 \%$ paraformaldehyde. Later the fixed cells were treated with $500 \mu \mathrm{M}$ and $1 \mathrm{mM}$ VK for $15 \mathrm{~min}$ at room temperature. The coverslips were then mounted on a glass slide, and images were captured in Leica confocal microscope.

\section{Concentration NMR}

NMR spectra have been recorded at 1, 3, 5, 7, $9 \mathrm{mg} / \mathrm{mL}$ in DMSO-d6, for VK. The study was carried out in the Avance neo $400 \mathrm{MHz}$ NMR Instrument. which has been performed to the check effects of concentration on pi-pi stacking of VK..$^{24,33-35}$

UV Visible

Fluorescence spectroscopy 


\section{Reference:}

1. Palmer, L. C.; Stupp, S. I., Molecular self-assembly into one-dimensional nanostructures. Accounts of chemical research 2008,41 (12), 1674-1684.

2. Whitesides, G. M.; Boncheva, M., Beyond molecules: Self-assembly of mesoscopic and macroscopic components. Proceedings of the National Academy of Sciences 2002,99 (8), 4769-4774.

3. Whitesides, G. M.; Mathias, J. P.; Seto, C. T., Molecular self-assembly and nanochemistry: a chemical strategy for the synthesis of nanostructures. Science 1991,254 (5036), 1312-1319.

4. Zhang, S., Fabrication of novel biomaterials through molecular self-assembly. Nature biotechnology 2003,21 (10), 1171-1178.

5. Habibi, Y.; Lucia, L. A.; Rojas, O. J., Cellulose nanocrystals: chemistry, self-assembly, and applications. Chemical reviews 2010,110 (6), 3479-3500.

6. Pope, M. T.; Müller, A., Introduction to polyoxometalate chemistry: from topology via selfassembly to applications. In Polyoxometalate Chemistry From Topology via Self-Assembly to Applications, Springer: 2001; pp 1-6.

7. Kushner, D., Self-assembly of biological structures. Bacteriological reviews 1969,33 (2), 302.

8. Whitesides, G. M.; Grzybowski, B., Self-assembly at all scales. Science 2002,295 (5564), 24182421.

9. Thiruvengadathan, R.; Korampally, V.; Ghosh, A.; Chanda, N.; Gangopadhyay, K.; Gangopadhyay, S., Nanomaterial processing using self-assembly-bottom-up chemical and biological approaches. Reports on Progress in Physics 2013,76 (6), 066501.

10. Cademartiri, L.; Bishop, K. J., Programmable self-assembly. Nature materials 2015,14 (1), 2-9. 11. Tang, Y.; Hill, E. H.; Zhou, Z.; Evans, D. G.; Schanze, K. S.; Whitten, D. G., Synthesis, selfassembly, and photophysical properties of cationic oligo ( $p$-phenyleneethynylene) s. Langmuir 2011,27 (8), 4945-4955.

12. Wong, K. M.-C.; Yam, V. W.-W., Self-assembly of luminescent alkynylplatinum (II) terpyridyl complexes: Modulation of photophysical properties through aggregation behavior. Accounts of chemical research 2011,44 (6), 424-434.

13. Saha, M. L.; Yan, X.; Stang, P. J., Photophysical properties of organoplatinum (II) compounds and derived self-assembled metallacycles and metallacages: Fluorescence and its applications. Accounts of Chemical Research 2016,49 (11), 2527-2539.

14. Li, Z.; Swihart, M.; Ruckenstein, E., Luminescent silicon nanoparticles capped by conductive polyaniline through the self-assembly method. Langmuir 2004,20 (5), 1963-1971.

15. Garnier, F.; Yassar, A.; Hajlaoui, R.; Horowitz, G.; Deloffre, F.; Servet, B.; Ries, S.; Alnot, P., Molecular engineering of organic semiconductors: design of self-assembly properties in conjugated thiophene oligomers. Journal of the American Chemical Society 1993,115 (19), 8716-8721.

16. Stone, D. A.; Tayi, A. S.; Goldberger, J. E.; Palmer, L. C.; Stupp, S. I., Self-assembly and conductivity of hydrogen-bonded oligothiophene nanofiber networks. Chemical Communications 2011,47 (20), 5702-5704.

17. Siram, R. B. K.; Tandy, K.; Horecha, M.; Formanek, P.; Stamm, M.; Gevorgyan, S.; Krebs, F. C.; Kiriy, A.; Meredith, P.; Burn, P. L., Synthesis and self-assembly of donor-acceptor-donor based oligothiophenes and their optoelectronic properties. The Journal of Physical Chemistry C 2011,115 (29), 14369-14376.

18. Giansante, C.; Carbone, L.; Giannini, C.; Altamura, D.; Ameer, Z.; Maruccio, G.; Loiudice, A.; Belviso, M. R.; Cozzoli, P. D.; Rizzo, A., Colloidal arenethiolate-capped PbS quantum dots: optoelectronic properties, self-assembly, and application in solution-cast photovoltaics. The Journal of Physical Chemistry C 2013,117 (25), 13305-13317. 
19. Jiang, G.; Hore, M. J.; Gam, S.; Composto, R. J., Gold nanorods dispersed in homopolymer films: optical properties controlled by self-assembly and percolation of nanorods. ACS nano 2012,6 (2), 1578-1588.

20. Sun, B.; Sirringhaus, H., Solution-processed zinc oxide field-effect transistors based on selfassembly of colloidal nanorods. Nano letters 2005,5 (12), 2408-2413.

21. Bâldea, I., A sui generis electrode-driven spatial confinement effect responsible for strong twisting enhancement of floppy molecules in closely packed self-assembled monolayers. Physical Chemistry Chemical Physics 2018,20 (36), 23492-23499.

22. Claessens, C. G.; Stoddart, J. F., $\pi-\pi$ Interactions in self-assembly. Journal of Physical Organic Chemistry 1997,10 (5), 254-272.

23. Gao, X.; Wang, Y.; Wang, X.; Guo, X.; Huang, J.; Yan, Y., Concentration-tailored selfassembly composition and function of the coordinating self-assembly of perylenetetracarboxylate. Journal of Materials Chemistry C 2017,5 (35), 8936-8943.

24. Boccia, A. C.; Lukeš, V.; Eckstein-Andicsová, A.; Kozma, E., Solvent-and concentrationinduced self-assembly of an amphiphilic perylene dye. New Journal of Chemistry 2020,44 (3), 892899.

25. Aoki, K. i.; Nakagawa, M.; Ichimura, K., Self-assembly of amphoteric azopyridine carboxylic acids: organized structures and macroscopic organized morphology influenced by heat, pH change, and light. Journal of the American Chemical Society 2000,122 (44), 10997-11004.

26. Hayward, D. W.; Chiappisi, L.; Teo, J. H.; Prévost, S.; Schweins, R.; Gradzielski, M., Neutralisation rate controls the self-assembly of pH-sensitive surfactants. Soft Matter 2019,15 (42), 8611-8620.

27. Korevaar, P. A.; Schaefer, C.; de Greef, T. F.; Meijer, E., Controlling chemical self-assembly by solvent-dependent dynamics. Journal of the American Chemical Society 2012,134 (32), 1348213491.

28. Chen, K.; Jiao, T.; Li, J.; Han, D.; Wang, R.; Tian, G.; Peng, Q., Chiral nanostructured composite films via solvent-tuned self-assembly and their enantioselective performances. Langmuir 2019,35 (9), 3337-3345.

29. Winfree, E.; Liu, F.; Wenzler, L. A.; Seeman, N. C., Design and self-assembly of twodimensional DNA crystals. Nature 1998,394 (6693), 539-544.

30. Yang, S.; Yan, Y.; Huang, J.; Petukhov, A. V.; Kroon-Batenburg, L. M.; Drechsler, M.; Zhou, C.; Tu, M.; Granick, S.; Jiang, L., Giant capsids from lattice self-assembly of cyclodextrin complexes. Nature communications 2017,8 (1), 1-7.

31. Kalsin, A. M.; Fialkowski, M.; Paszewski, M.; Smoukov, S. K.; Bishop, K. J.; Grzybowski, B. A., Electrostatic self-assembly of binary nanoparticle crystals with a diamond-like lattice. science 2006,312 (5772), 420-424.

32. Kakuno, M.; Izawa, S.; Takemoto, T.; Tanabe, Y., UTILIZATION OF CHLOBENTHIAZONE AND BENAZOLIN-ETHYL AS 4-SUBSTITUTED 2 (3H)-BENZOTHIAZOL-2-ONE SCAFFOLDS. HETEROCYCLES 2018,96 (11), 1925-1939.

33. Shao, C.; Grüne, M.; Stolte, M.; Würthner, F., Perylene Bisimide Dimer Aggregates: Fundamental Insights into Self-Assembly by NMR and UV/Vis Spectroscopy. Chemistry-A European Journal 2012,18 (43), 13665-13677.

34. Nişancı, B.; Daştan, A.; Bozdemir, Ö. A., Aromatic stacking of a perylenetetracarboxylic tetraester: Self-assembly in both water and chloroform. Tetrahedron Letters 2018,59 (39), 35583562.

35. Li, A. D.; Wang, W.; Wang, L. Q., Folding versus self-assembling. Chemistry-A European Journal 2003,9 (19), 4594-4601.

36. Ge, Y.; Wen, Y.; Liu, H.; Lu, T.; Yu, Y.; Zhang, X.; Li, B.; Zhang, S.-T.; Li, W.; Yang, B., A key stacking factor for the effective formation of pyrene excimer in crystals: Degree of $\pi-\pi$ overlap. Journal of Materials Chemistry C 2020,8 (34), 11830-11838. 